\title{
Shoreline fish distribution in Italian lakes assessed by electrofishing
}

Silvia Galafassi (iD , Paolo Sala \& Pietro Volta (D)
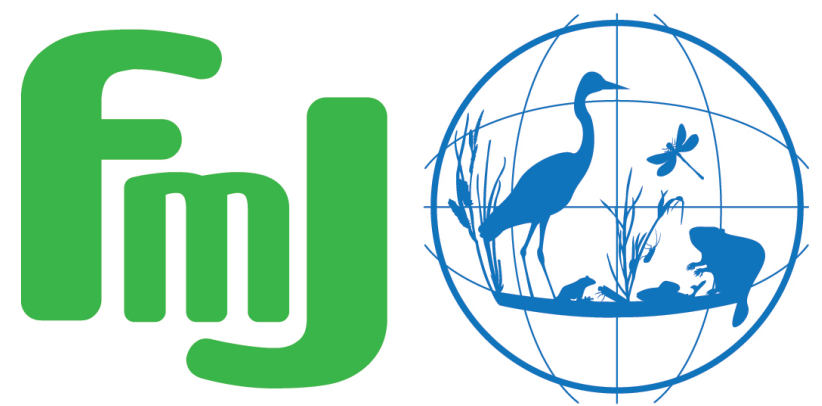

Freshwater Metadata Journal 



\title{
Shoreline fish distribution in Italian lakes assessed by electrofishing
}

\author{
Silvia Galafassi ${ }^{1}$ (iD), Paolo Sala ${ }^{1} \&$ Pietro Volta $^{1}$ (iD \\ 1 CNR IRSA Water Research Institute, Verbania Pallanza, Italia; corresponding author: pietro.volta@irsa.cnr.it
}

Please cite this paper as follows: Galafassi, S, Sala, P \& Volta, P, 2019. Shoreline fish distribution in Italian lakes assessed by electrofishing. Freshwater Metadata Journal 42: 1-5.

https://doi.org/10.15504/fmj.2019.42

Received: 2019-02-22 / Published: 2019-02-22

\section{Keywords}

fish biodiversity, electrofishing, lakes, reservoirs

\section{Short description of the dataset/summary}

The dataset includes data on fish species distribution along the shoreline of 25 Italian lakes and reservoirs achieved by point abundance standardised electrofishing. Data were obtained between 2007 and 2014. The database offers a detailed description of the biodiversity of fish communities in the Italian regions of Piedmont, Lombardy, Trentino-Alto Adige and Sardinia including both natural lakes and reservoirs.

\section{Short description of the dataset/summary (original/national language)}

Il dataset include le informazioni sulla distribuzione della fauna ittica nella zona litorale di 25 laghi italiani. Le informazioni raccolte sono basate su campionamenti effettuati con elettrostorditore da una barca, condotti seguendo la riva di 25 bacini italiani.

\section{General information}

dataset entry ID: $\quad$ FWM_20

\section{name of the dataset:}

full name of the dataset: Shoreline fish distribution in Italian lakes assessed by electrofishing

full name of the datast (original/national language):

type of dataset: species distribution data

data type: point data/observation data

science keywords according to GCMD:

topic:

Biosphere, Biological Classification, Climate Indicators, Terrestrial 
Hydrosphere

ISO topic category according to ISO 19115:

Biota, Environment, Inland Waters

INSPIRE keywords according to GEMET:

Species distribution

own science keywords:

freshwater fish; Italy; lakes; non native fish species; PASE electrofishing

related project:

LIFE+ Inhabit, WISER, CENSIMENTO DELLA FAUNA ITTICA DEI

LAGHI SUDALPINI

funding:

INHABIT - LIFE08 ENV/IT/000413.

WISER - European Union, 7th Framework Programme, Theme 6

(Environment including Climate Change) (contract No. 226273).

CENSIMENTO DELLA FAUNA ITTICA DEI LAGHI SUDALPINI -

REGIONE LOMBARDIA.

\section{Technical and administrative specifications}

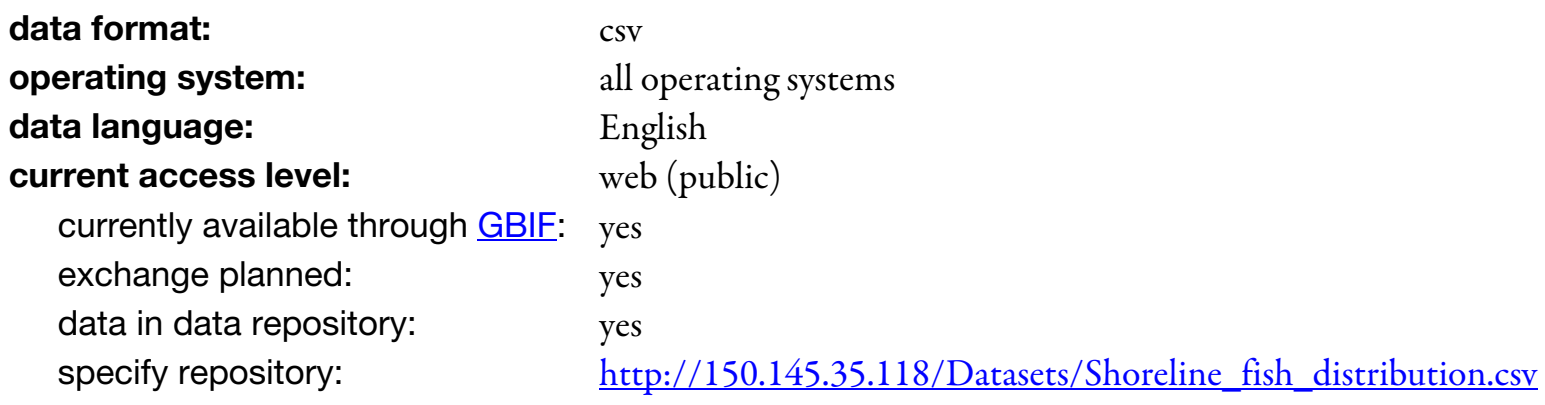

\section{Do you plan to publish the data on the Freshwater Biodiversity Data Portal:}

$\begin{array}{ll}\begin{array}{l}\text { update level: } \\ \text { documentation: } \\ \text { type: }\end{array} & \text { completed } \\ \text { language: } & \text { manual } \\ & \text { English } \\ \text { contact details: } & \\ \text { metadata contact person: } & \\ \text { first, last name: } & \text { Pietro Volta } \\ \text { phone: } & +390323518335 \\ \text { email: } & \text { p.volta@cnr.it } \\ \text { institution: } & \text { CNR Water Research Institute } \\ \text { address: } & \text { Largo Tonolli } 50 \\ \text { postal code, city: } & 28922 \text { Verbania } \\ \text { province, state: } & \text { VB } \\ \text { country } & \text { Italy } \\ \text { web address: } & \underline{\text { http://www.irsa.cnr.it/ }} \\ \text { technical contact person: } & \\ \text { first, last name: } & \text { Silvia Galafassi } \\ \text { phone: } & +390323518357 \\ \text { email: } & \text { silvia.galafassi@gmail.com } \\ \text { scientific contact person: } & \\ \text { first, last name: } & \text { Pietro Volta } \\ \text { phone: } & +390323518335\end{array}$


email:

p.volta@cnr.it

\section{Intellectual property rights and citation}

dataset creator (data compiler):

contact name:

contact email:

Pietro Volta

contact institution:

p.volta@cnr.it

Water Research Institute IRSA - Consiglio Nazionale delle Ricerche

data contributors to/owners of this dataset:

single

criteria for using this dataset: The dataset is publicly available (data portal, data archive) and can be used without restrictions, but dataset creator/data contributors must be informed prior to publication. Data must be acknowledged and cited correctly.

citation of this dataset:

author(s):

Volta, P. \& Galafassi, S.

title and journal (name, number, pages):

Database on shoreline fish distribution in italian lakes assessed by electrofishing.

year:

2018

doi:

https://doi.org/10.15468/pq0enb

citation of the metadata:

author(s):

Galafassi, S, Sala, P \& Volta, P

title and journal (name, number, pages):

Shoreline fish distribution in Italian lakes assessed by electrofishing. Freshwater

Metadata Journal 42: 1-5

year: 2019

doi: $\quad$ https://doi.org/10.15504/fmj.2019.42

\section{General data specifications}

regional coverage of the dataset:

spatial extent of the dataset:

national

continents:

Europe

spatial extent (bounding coordinates):

southernmost latitude $\left[^{\circ}\right.$ ]:

40.553089

northernmost latitude $\left[^{\circ}\right]$ :

46.756744

westernmost longitude $\left[^{\circ}\right]$ :

7.125792

easternmost longitude $\left[^{\circ}\right]$ :

12.085461

minimum altitude:

43 metres

maximum altitude:

2275 metres

countries:

Europe: Italy

world climatic regions according to Köppen:

Group C: temperate/mesothermal climates

Group D: continental/microthermal climate

Group $\mathrm{H}$ : alpine climates

European ecoregions according to Illies (WFD):

Italy, Corsica and Malta (ER3)

ecosystem type:

lakes/ponds

covered timeframe:

$2007-2014$ 


\section{Site specifications}

coordinate system/grid data:

datum (e.g. WGS84):

number of sites:

exact number of sites:

\section{Biological data}

biological data origin:

comments:

organism group addressed: latitude/longitude, format: DD

projected, UTM

WGS84

$<100$

25

from sampling,

INHABIT - LIFE08 ENV/IT/000413. WISER - European Union, 7th Framework Programme, Theme 6 (Environment including Climate Change) (contract No. 226273). CENSIMENTO DELLA FAUNA ITTICA DEI LAGHI SUDALPINI - REGIONE LOMBARDIA

fish

\section{Sample resolution}

fish:

\section{taxonomic resolution:}

level: species

percentage of species level data: 100

\section{taxonomic coding:}

taxalist according to:

Kottelat \& Freyhof

reference(s):

Kottelat, M. \& Freyhof, J., 2007. Handbook of European freshwater fishes.

Publications Kottelat, Cornol and Freyhof, Berlin. 646 pp.

\section{sample specifications:}

specification of method(s) used for sampling and sorting:

The electrofishing device was a built-in-frame EL64GII (Scubla Acquaculture, $7,000 \mathrm{~W}, 600 \mathrm{~V}$, DC current) set up with a copper cathode (width $2.5 \mathrm{~cm}$, length $300 \mathrm{~cm}$ ) and a steel ring anode (thickness $0.8 \mathrm{~cm}$, diameter $50 \mathrm{~cm}$ ). The Point Abundance Sampling Electrofishing (PASE) method (Copp \& Garner, 1995; ISPRA, 2014) was used in which the anode is dipped for $20 s$ at each sampling point. The number of points depended on lake size and habitat complexity.

reference(s):

Copp, G. H. \& Garner, P., 1995. Evaluating microhabitat use of fish larvae and juveniles with point abundance sampling. Folia Zoologica 44: 145-158.

ISPRA, 2014. Metodi biologici per le acque superficiali interne. Manuali e linee guida 111/2014. Rome, Italy. ISBN 978-88-448-0651

\section{Other specifications}

\section{GIS layers, shape files related to the dataset:}




\section{quality control procedures:}

Were any quality control procedures applied to your dataset?

no

\section{References}

Copp, G. H. \& Garner, P., 1995. Evaluating microhabitat use of fish larvae and juveniles with point abundance sampling. Folia Zoologica 44: 145-158.

ISPRA, 2014. Metodi biologici per le acque superficiali interne. Manuali e linee guida 111/2014. Rome, Italy. ISBN 978-88-448-0651

Kottelat, M. \& Freyhof, J., 2007. Handbook of European freshwater fishes. Publications Kottelat, Cornol and Freyhof, Berlin. 646 pp. 